\title{
Quantitative Detection of Aminoglutethimide by Electrochemical Surface Enhanced Raman Spectroscopy
}

\author{
Z.C. Zhu ${ }^{1}$, W. V. Espulgar ${ }^{1}$, H. Yoshikawa ${ }^{1}$, M. Saito ${ }^{1,2}$, E. Tamiya ${ }^{1}$ \\ ${ }^{1}$ Osaka University, 2-1 Yamadaoka, Suita, Osaka, JAPAN \\ ${ }^{2}$ Advanced Photonics and Biosensing Open Innovation Laboratory, 2-1 Yamadaoka, Suita, Osaka, \\ JAPAN \\ zhuzicheng@ap.eng.osaka-u.ac.jp
}

\begin{abstract}
:
In this work, we have investigated the detection of aminoglutethimide (AGI) drug based on its adsorption on the SERS-active screen-printed electrode employing the Electrochemical Surface-Enhanced Raman Spectroscopy (E-SERS) technology. E-SERS spectra exhibits the different absorption of AGI onto the substrate. At the $-400 \mathrm{mV}$ of applied potential, the E-SERS peaks of $1147 \mathrm{~cm}^{-1}$ shows the strongest intensity among the other peaks. The limit of detection (LOD) is $40 \mathrm{ng} / \mathrm{mL}$ and the square $R$ of linear curve is 0.98 . This work aims to optimize the trace detection of this drug with possible application in the deterrence and prevention of dopant usage and for point-of-care diagnostics (POCD).
\end{abstract}

Key words: aminoglutethimide, electrochemical SERS, anti-doping, point-of-care diagnostics.

\section{Introduction}

Aminoglutethimide (AGI) is an aromatase inhibitor which is used clinically for the treatment of hormone-dependent metastatic breast cancer [1]. However, it can also be used as dopant and thus the consumption of AGI by sportsmen in official competitions is banned by the World AntiDoping Agency [2].

The electrochemical SERS (E-SERS) sensors have been rapidly developed and widely used in a range of applications due to its enhanced chemical detection. Benefited from the adjustable potentials on the electrode, the orientation of the molecules on the substrate can be changed which affects the intensity of SERS signal. Both chemical and physical enhancements of SERS can be influenced to a certain extent by applying an electrode potential. In this study, the electrochemical SERS sensor offers the advantages of enhanced signal intensity and more selectivity to the $A G I$ molecule.

\section{Methodology}

The screen-printed electrode (SPE) consists of a reference electrode $(\mathrm{Ag} / \mathrm{AgCl})$, a counter electrode (carbon) and a working electrode (carbon or gold) shown in Fig. 1.

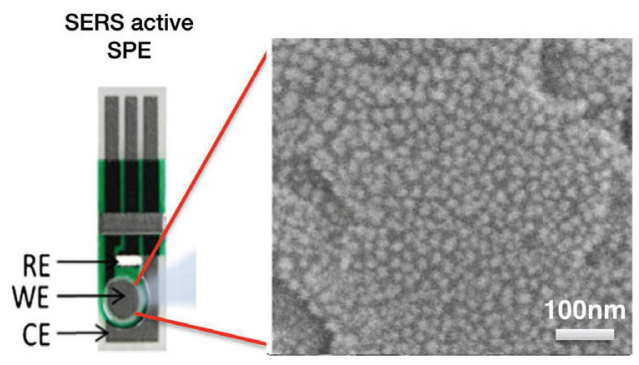

Fig. 1. The photo of SERS active SPE used in this work which is composed of a working electrode (WE), a counter electrode (CE) and a reference electrode (RE). SEM image of silver-deposited carbon working electrode. The diameters of silver nanoparticles are approximately ranging from $20 \mathrm{~nm}$ to $30 \mathrm{~nm}$.

The SPEs were mounted to a customized mask so that only the areas of the working electrode were exposed for sputtering deposition by in-line sputtering machine (V0005, Optorun, Japan). The silver nanoparticles were deposited onto the working electrode of the screen-printed electrode. The target power was set to $250 \mathrm{~kW}$ for the sputtering process. The deposition time of sputtering process was $12 \mathrm{~s}$. To avoid melting the carbon electrode and the plastic masks, the 

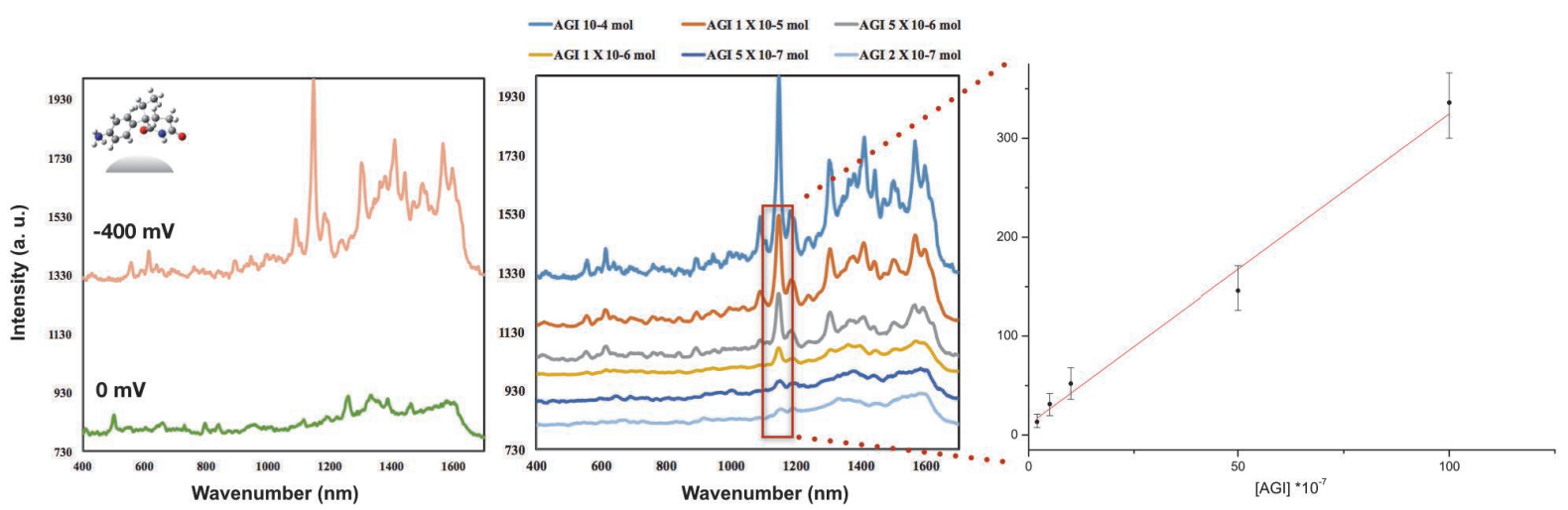

Fig.2. Left graph: Comparison of the SERS (0 mV) and E-SERS (-400 mV vs Ag/AgCl) spectra of $0.1 \mathrm{mM} \mathrm{AGI}$ solution. Middle graph: E-SERS spectra of AGI at the applied potential of $-400 \mathrm{mV}$ vs Ag/AgCl with enhanced peak signal at $1147 \mathrm{~cm}-1$. Right graph: Calibration curve of different drug concentrations of AGI with good linear dependence (0.98). Error bar represents standard deviation where $n=4$.

whole sputtering process was done under 50 degrees centigrade. The $30 \mu \mathrm{L}$ of solution was placed on the substrate. The Raman spectra and Raman images were obtained with a laser Raman confocal microscope (RAMAN-11, Nanophoton, Japan) equipped with an Olympus 20x, water-immersion objective lens. The sample was illuminated with a line-shaped laser (532 $\mathrm{nm}$ was about $2.8 \mathrm{~mW} /$ line (400 pixels) focused through the objective lens and Raman scattering from the samples was collected by the same objective lens and guided to a spectrophotometer with a slit width of $15 \mu \mathrm{m}$. The Raman signal was diffracted by a 600grooves $/ \mathrm{mm}$ grating (spectra resolution, $1.6 \mathrm{~cm}$ $\left.{ }^{1}\right)$ and was detected by an air-cooled CCD camera $\left(-70{ }^{\circ} \mathrm{C}\right)$. Raman spectra were obtained from $1.25 \mu \mathrm{m} \times 167 \mu \mathrm{m}(3 \times 400$ pixels $)$ area. The potentiostat system was a USB portable device. By applied different potentials (from 0 to $1000 \mathrm{mV}$ with the increment of $-200 \mathrm{mV}$ ) to the SERS-active substrate, different absorption of the AGI molecule onto the silver nanoparticles brought the benefits of enhanced signals and more selective SERS peaks.

\section{Result}

The AGI was successfully detected on the ESERS chip shown in Fig. 2. Compared with the SERS spectra (without applied potential), the signal intensities of E-SERS spectra were enhanced at the applied potential of $-400 \mathrm{mV}$ vs $\mathrm{Ag} / \mathrm{AgCl}$. The $\mathrm{C}-\mathrm{H}$ in-plane bending of the AGI (corresponding to the wavenumber of $1147 \mathrm{~cm}^{-1}$ ) showed the strongest peak intensity among the other peaks at the $-400 \mathrm{mV}$ applied potential. The limit of detection (LOD) is $2 \times 10^{-7}(40 \mathrm{ng} / \mathrm{ml})$ and the square $\mathrm{R}$ of linear fitting curve equivalent to 0.98 ; indicating a good linear relationship.

\section{Conclusion}

E-SERS sensor successfully detects the AGI molecule at the low level of concentration. A linear dependence of different concentrations in the [AGI] range occurs from $2 \times 10^{-7}$ to $1 \times 10^{-5}$, which corresponds to the range from $40 \mathrm{ng} / \mathrm{ml}$ to $2 \mu \mathrm{m} / \mathrm{ml}$. Besides the enhanced signal, more selective peak signals of the AGI can be observed by the applied different potentials which exhibit the possibility for SERS technique applying to some complex case, even for clinical application in the future.

\section{References}

[1] A. Elbashir, F. Suliman, B. Saad and H. Enein, Determination of aminoglutethimide enantiomers in pharmaceutical formulations by capillary electrophoresis using methylated-cyclodextrin as a chiral selector and computational calculation for their respective inclusion complexes, Talanta, 77 , 1388-1393 (2009); doi:10.1016/j.talanta. 2008.09.029.

[2] I. Lorenzo, S. Cortes and J. V. Ramos, Trace detection of aminoglutethimide drug by surface-enhanced Raman spectroscopy: a vibrational and adsorption study on gold nanoparticles, Anal. Methods, 3, 1540-1545 (2011); doi:10.1039/c1ay05032j. 